\title{
The Energy of Liposome Patch Adhesion to the Pipette Glass Determined by Confocal Fluorescence Microscopy
}

J. Phys. Chem. Lett., 2016, 7 (22), pp 4530-4534

DOI: 10.1021/acs.jpclett.6b02027

Yoshitaka Nakayama ${ }^{1}$, Radomir I. Slavchov ${ }^{2,3}$, Navid Bavi $^{1,4}$, Boris Martinac ${ }^{1,4}$

${ }^{1}$ Mechanosensory Biophysics Laboratory, Victor Chang Cardiac Research Institute, Darlinghurst, New South Wales 2010, Australia

${ }^{2}$ Department of Physical Chemistry, Sofia University, 1 J. Bourchier Blvd., Sofia 1126, Bulgaria

${ }^{3}$ Department of Chemical Engineering and Biotechnology, Cambridge University, Pembroke Street, New Museums Site, CB2 3RA Cambridge, United Kingdom

${ }^{4}$ St. Vincent's Clinical School, University of New South Wales, Darlinghurst, New South Wales 2052, Australia 


\begin{abstract}
The formation of the gigaseal in the patch-clamp technique is dependent on the adhesion between the cell or liposome membrane and the glass pipette. The adhesion results in a capillary force causing creep of the patch membrane upward the pipette. The membrane can be immobilized by counteracting the capillary force by positive pressure applied to the patch pipette. We use this phenomenon to develop a method for static measurement of the adhesion free energy of the lipid bilayer to the glass. Confocal fluorescent microscopy is used to track the bilayer creep inside the pipette and measure the immobilization pressure at various salt concentrations and $\mathrm{pH}$. The adhesion energy is simply related to this pressure. For the studied phospholipid bilayers, its values were in the range $0.3-0.7 \mathrm{~mJ} / \mathrm{m}^{2}$, increasing with salt concentration and having a maximum as function of $\mathrm{pH}$. This method offers a more precise way for the measurement of bilayer-glass adhesion energy in patch clamp experiments than dynamic methods.
\end{abstract}

\title{
TOC GRAPHICS
}

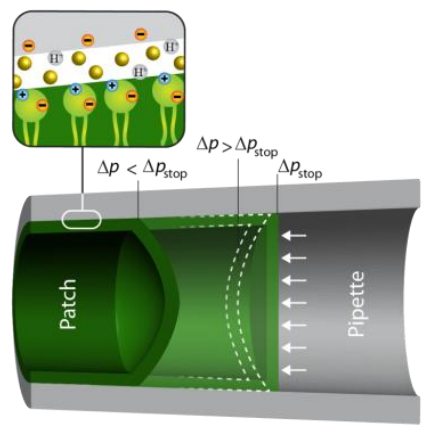

KEYWORDS (6/6 keywords)

gigaseal, patch clamp, adhesion tension, azolectin, lipid bilayer, capillary effect

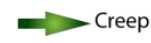




\section{Main text}

Cell adhesion is an important process cells use to interact with each other and attach to a substrate [1,2]. For many years, biophysicist have studied it as well as tried to manipulate it [3,4]. Cells and bacteria adhere to surfaces using a variety of mechanisms, which are subject of intensive research [1,2]. Many basic experimental procedures involving biomembranes require tight control of adhesion. For instance, the adhesion of cells onto solid surfaces is widely used as a method for extraction of plasma membrane $[5,6]$. The patch-clamp is another technique, which depends on the existence of adhesion forces promoting the tight contact between the membrane and the patch pipette ensuring the formation of the gigaseal between the bilayer and the pipette glass $[7,8]$. A number of studies by patch-clamp electrophysiologists focused on estimation of the lipid-glass adhesion energy and on the analysis of its conjugation with the membrane tension that activates mechanosensitive channels in a patch membrane $[7,9,10]$.

The basic characteristic of the adhesion of a cell or a liposome to the glass is their adhesion energy $\sigma_{\text {adh. }}$ It is defined as the difference between the free surface energy of the free membrane and glass surfaces $\left(\sigma^{\mathrm{M}}+\sigma^{\mathrm{G}}\right)$ and the free energy of the membrane adhered to the glass $\left(\sigma^{\mathrm{M}}+\sigma^{\mathrm{G}}\right.$ $\left.-\sigma_{\text {adh }}\right)$. Several methods for measurement of the adhesion energy have been proposed. The classical one [11] is based on Young's balance at the 3 phase contact (3PC) (Figure S1 in the supporting information):

$$
\sigma_{\mathrm{adh}} / \sigma^{\mathrm{M}}=1+\cos \theta_{\mathrm{eq}}
$$

Here $\theta_{\mathrm{eq}}$ is the equilibrium contact angle of the adhered cell or liposome. Contact angle measurements can therefore be used for the determination of $\sigma_{\mathrm{adh}}$, provided that the membrane tension $\sigma^{\mathrm{M}}$ is known. However, careful measures must be taken to eliminate 3PC line creep since the dynamic contact angle (advancing or receding) vary greatly with creep velocity [12]. The shape of a liposome adhered onto a solid must relax to the equilibrium contact angle in order Eq. (1) to be applicable. In a capillary, the establishment of this equilibrium is complicated. A meniscus climbing into a vertical capillary has a dynamic contact angle different from $\theta_{\text {eq }}$ until Jurin's equilibrium capillary uphold height is reached [13]. In a horizontal capillary, the meniscus moves with non-zero velocity until the whole capillary is filled - the contact angle is function of this velocity and $\theta_{\text {eq }}$ may never be actually observed. 
Several other methods for determination of $\sigma_{\text {adh }}$ exist. Smith et al. proposed a method based on the balance between tether formation and adhesion [14]. In another study [15], the velocity $v_{L} \equiv$ $\mathrm{d} L / \mathrm{d} t$ of creep of patches in a patch-clamp pipette was related to the driving force (adhesion and/or applied pressure) of the creep:

$$
v_{L}=\left(\sigma_{\mathrm{adh}}-1 / 2 R_{\mathrm{c}} \Delta p\right) / k_{\eta}
$$

Here $L$ is the length of the glass pipette that has bilayer adhered (Figure 1); $R_{\mathrm{c}}$ is the radius of the pipette at the 3PC; $\Delta p$ is the applied pressure (suction pressure in the capillary corresponds to negative sign of $\Delta p$ ), and $k_{\eta}$ is a friction coefficient related to the mechanism of creep and the geometry of the pipette [15]. Eq. (2) was used to determine $\sigma_{\text {adh }}$ together with $k_{\eta}$ for azolectin liposomes in contact with glass [15]. This was done by measuring first $v_{L}$ at $-\Delta p \gg 2 \sigma_{\mathrm{adh}} / R_{\mathrm{c}}$ (which allows the determination of $k_{\eta}$ ) and then $v_{L}$ at $\Delta p=0$ (once $k_{\eta}$ is known, this yields $\sigma_{\text {adh }}$ ). This method yielded reasonable values for $\sigma_{\text {adh }}$, but has nevertheless disadvantages - most importantly, various kinetic effects such as non-linear force-velocity dependence $[12,13]$ and dome bulging may result in a dependence of $k_{\eta}$ on $v_{L}$, ultimately leading to inaccurate results.

In addition, there are several widely used methods that measure quantities directly related to the adhesion energy. For example, Priel et al. [16] utilized AFM to measure glass-membrane interactions, which are proportional to $\sigma_{\text {adh. }}$. The traditional measure of cell adhesion - the number of adhered cells $[3,5]-$ is proportional to the Boltzmann factor $\exp \left(-a \sigma_{\mathrm{adh}} / k_{\mathrm{B}} T\right)$, where $a$ is the cell-solid contact area. In another method, an apparent adhesion energy $\sigma_{\mathrm{adh}, \mathrm{n}}$ is determined as a force normal to the glass surface, balancing the normal component $\sigma^{\mathrm{M}} \sin \theta$ of the membrane tension in Figure S1. From the assumed balance $\sigma^{\mathrm{M}} \sin \theta=\sigma_{\mathrm{adh}, \mathrm{n}}$, a value of $\sigma_{\mathrm{adh}, \mathrm{n}}$ follows $[7,17]$. This treatment neglects the elastic answer of the solid substrate which is actually believed to be the major normal response to $\sigma_{\text {adh,n }}[18]$. In addition, the relation between $\sigma_{\text {adh,n }}$ and $\sigma_{\text {adh }}$ is not straightforward ( $\sigma_{\mathrm{adh}, \mathrm{n}}=-\lambda / R_{\mathrm{c}}$ where $\lambda$ is the line tension, which is the linear Gibbs excess of the adhesion energy $\left.\sigma_{\text {adh }}[19]\right)$. 


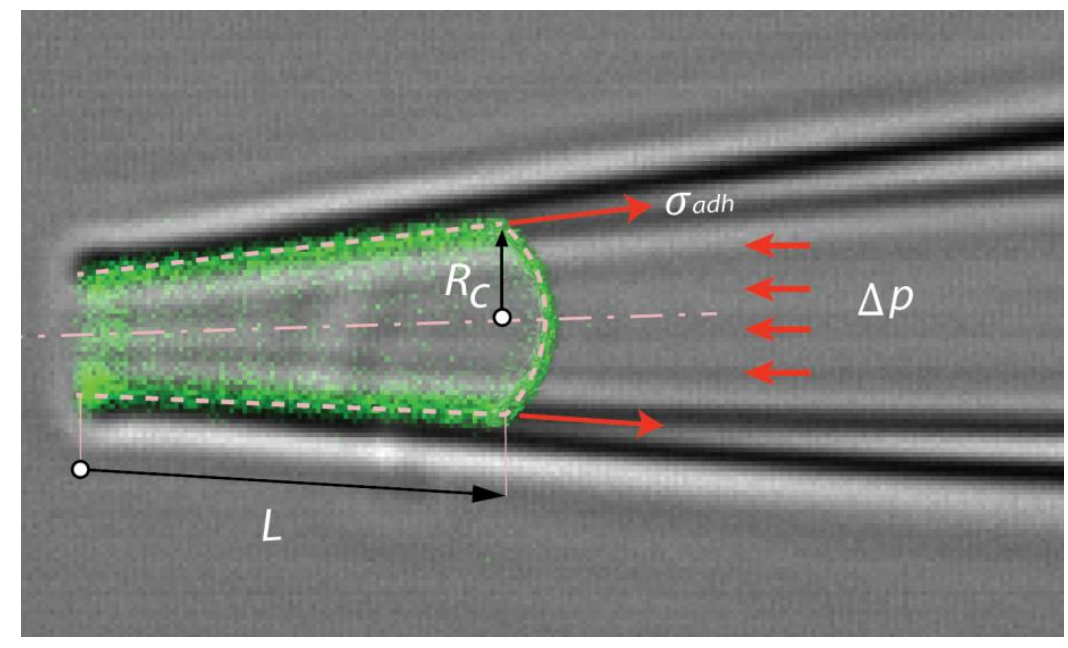

Figure 1 Geometry of a liposome patch in a typical capillary.

The dynamic complications with the force balance at the 3PC line result in significant variations in the experimental results for $\sigma_{\mathrm{adh}}$ in the literature. For example, Ursell et al. [10] reported that the adhesion energy they determined for lipid bilayers on glass could vary by a factor of 2-3 for the same lipid mixture and glass. The difference between their $\sigma_{\mathrm{adh}}$ and the values from the dynamic method in Ref. [15] is nearly one order of magnitude, although the studied bilayers are similar. Therefore, a robust method is needed that does not have these issues.

It was observed in Ref. [15] that from Eq. (2) it follows that the patch can be immobilized by compensating the adhesion force by applying counteracting positive pressure $\Delta p$ after the gigaseal is formed between the patch and the pipette - according to Eq. (2), the creep velocity is zero when

$$
\Delta p=2 \sigma_{\mathrm{adh}} / R_{\mathrm{c}} \quad\left(\equiv \Delta p_{\text {stop }}\right), \quad \text { i.e. } \quad \sigma_{\mathrm{adh}}=1 / 2 R_{\mathrm{c}} \Delta p_{\text {stop }}
$$

This equation can be obtained easily by balancing the force acting on the dome $\operatorname{rim}\left(2 \pi R_{\mathrm{c}} \sigma_{\mathrm{adh}}\right)$ with the external force applied to the patch $\left(\pi R_{\mathrm{c}}^{2} \Delta p\right)$. Eq. (3) can be utilized in a static technique for measuring $\sigma_{\mathrm{adh}}$, in which the pressure in the patch-clamp capillary $\Delta p$ is varied until $v_{L}=0$ (mechanical compensation of the capillary force), with obvious advantages over the dynamic method of Ref. [15].

In this study, we demonstrate the implications of this new method by measuring the adhesion energy of a bilayer to glass for relatively simple systems. The procedure we developed is described in the Materials \& Methods section. We applied this technique to measure $\sigma_{\text {adh }}$ of azolectin liposomes to borosilicate glass pipettes (Figure 1 gives the approximate geometry). Two series of 
experiments were performed: one in which the ionic strength of the saline solutions has been varied at fixed $\mathrm{pH}$, and another with varying $\mathrm{pH}$.

The measurements showed that the adhesion energy depends only weakly on the electrolyte concentration, cf. Figure $2 \mathrm{~B}$ and Table S1: the increase of $\mathrm{KCl}$ from $C_{\mathrm{KCl}}=100$ to $500 \mathrm{mM}$ (with $5 \mathrm{mM}$ HEPES-KOH buffer and $40 \mathrm{mM} \mathrm{MgCl} 2$ in addition, used as usually [20] to facilitate the gigaseal formation) results into a rather small increase of $\sigma_{\mathrm{adh}}$ from 0.5 to $0.7 \mathrm{~mJ} / \mathrm{m}^{2}$ (at precision $\left.\pm 0.1 \mathrm{~mJ} / \mathrm{m}^{2} ; \mathrm{mJ} / \mathrm{m}^{2} \equiv \mathrm{mN} / \mathrm{m}\right)$. This finding agrees with the results of Priel et al. for the adhesion force acting between membrane and glass [16] - these authors observed unexpectedly little effect from $\mathrm{NaCl}$ in the concentration range 0.1-1000 mM. The weak dependence on electrolyte concentration can be qualitatively explained within the framework of the DLVO theory, under the hypothesis that the seal (the aqueous film between the glass and the membrane) is very thin, having thickness $h$ smaller than the Debye length $L_{D}$ of the solution. The formulae for the electrostatic repulsion in an asymmetric film (aqueous solution of 1:1 electrolyte) of thickness $h<L_{\mathrm{D}}$ with surfaces of constant surface charge density are summarized in Ref. [15] (eqs A18-A20). In S4, we derive the respective series for the electrostatic contribution $\sigma_{\mathrm{el}}$ to the adhesion energy at $h \rightarrow 0$, under the assumption for relatively high surface potentials $\phi$ :

$$
\sigma_{\mathrm{el}} \approx-2 k_{\mathrm{B}} T\left(\Gamma^{\mathrm{M}}+\Gamma^{\mathrm{G}}\right)+\mathrm{O}\left(\phi^{0}\right)+\mathrm{O}\left(h^{1}\right)
$$

The leading term in this expansion is concentration-independent, as the adsorbed charge densities $\Gamma^{\mathrm{M}}$ at the membrane and $\Gamma^{\mathrm{G}}$ at the glass do not change significantly with the $\mathrm{KCl}$ concentration $C_{\mathrm{KCl}}$ (which is physically equivalent to $\Gamma^{\mathrm{M}} \& \Gamma^{\mathrm{G}}$ being independent of $h$ ). The increase of $C_{\mathrm{KCl}}$ leads to an increase of $\sigma_{\mathrm{adh}}$ through second-order effects only. Yet, even these second-order effects are large enough to result in a decrease (in absolute values) of the repulsive $\sigma_{\mathrm{el}}$ by about $0.5 \mathrm{~mJ} / \mathrm{m}^{2}$ at the highest concentration compared to the lowest, cf. Table S1 in S3. The observed increase of the total energy $\sigma_{\mathrm{adh}}$ with $C_{\mathrm{KCl}}$ is only $0.1-0.2 \mathrm{~mJ} / \mathrm{m}^{2}$ instead. A probable reason for the difference between the two is that the electrolyte also screens [21] the attractive dispersion glass-membrane interaction, leading to decrease of the respective positive contribution $\sigma_{\mathrm{vdW}}$ to $\sigma_{\mathrm{adh}}$. If the addition of $400 \mathrm{mM} \mathrm{KCl}$ decreases the van der Waals attraction by $\sim 20 \%$ (proportionally to the numbers in Ref. [21]), and if $\sigma_{\mathrm{adh}}$ is of the order of $0.2-0.5 \times \sigma_{\mathrm{vdW}}$ [15], then the decrease of $\sigma_{\mathrm{adh}}$ due to this effect would be $\sim 0.2-0.6 \mathrm{~mJ} / \mathrm{m}^{2}$, compensating a significant part of the respective decrease of $\left|\sigma_{\mathrm{el}}\right|$. 
Another factor is that the electrolyte weakens the $\mathrm{Mg}^{2+}$ bridges binding glass and membrane [22, 23], but the magnitude of this effect cannot be estimated easily.

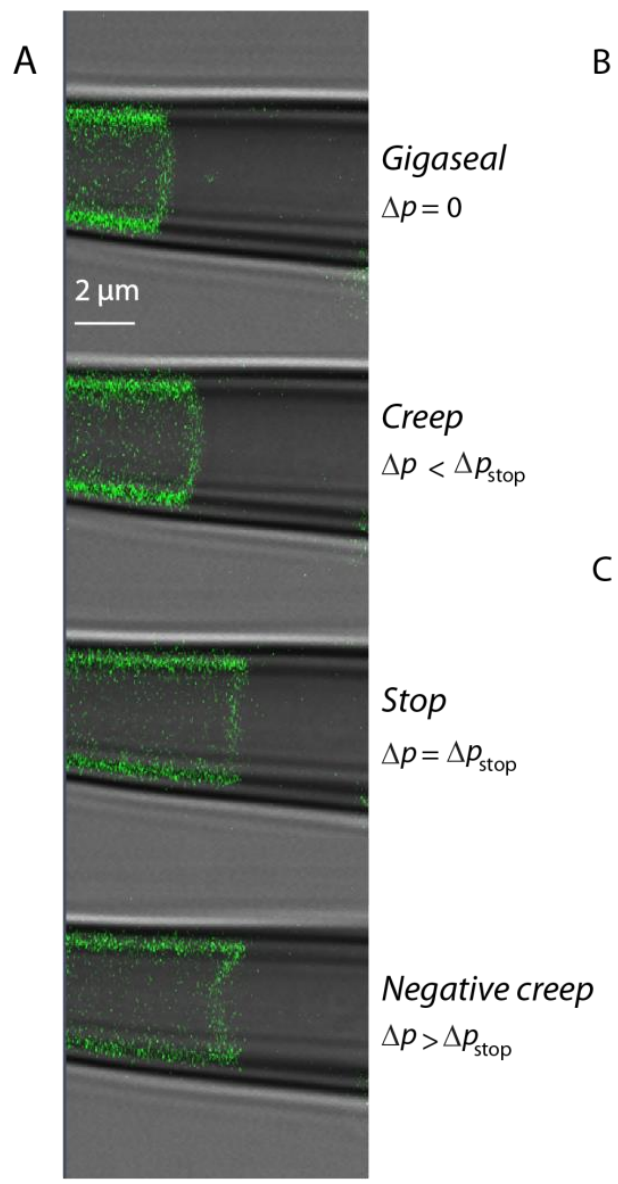

B

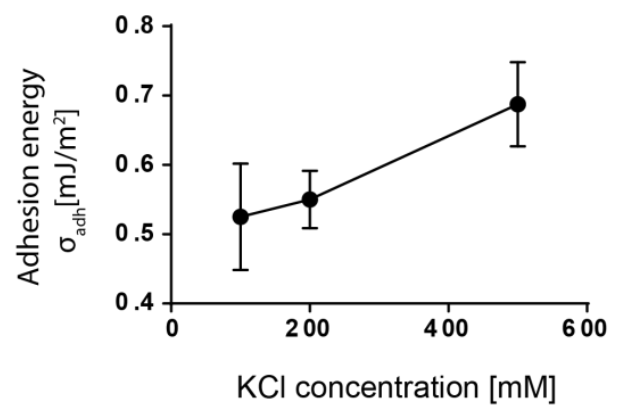

C

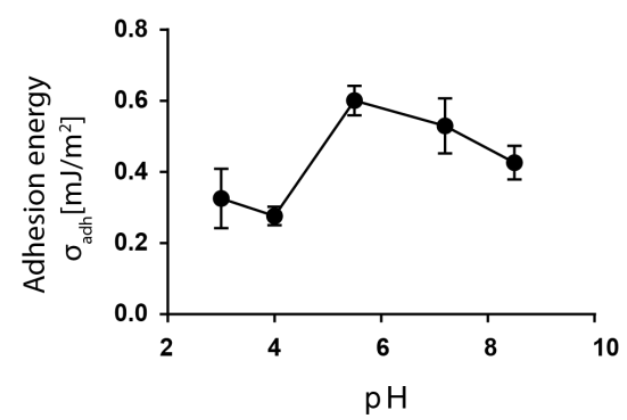

Figure 2 (A) Patch creep inside the pipette. Positive pressure $\Delta p$ is applied to the outer wall of the patch to counteract the adhesion-driven membrane creep. The pressure is varied until the patch is immobilized at $\Delta p=\Delta p_{\text {stop }}$; the adhesion energy is then determined via Eq. (3). (B) Effect of the concentration of $\mathrm{KCl}$ on the adhesion energy $\sigma_{\mathrm{adh}}$ of azolectin bilayer to the glass $\left(40 \mathrm{mM} \mathrm{MgCl}_{2}\right.$, $5 \mathrm{mM}$ HEPES-KOH, $\mathrm{pH}=7.2)$. (C) Effect of $\mathrm{pH}$ on $\sigma_{\mathrm{adh}}(100 \mathrm{mM} \mathrm{KCl}, 40 \mathrm{mM} \mathrm{MgCl} 2,5 \mathrm{mM}$ HEPES-KOH, pH adjusted by $\mathrm{HCl}$ or $\mathrm{KOH})$. The values are mean $\pm \mathrm{SEM}$.

In the second series of experiments, $\mathrm{pH}$ was varied at fixed ionic strength - the results for $\sigma_{\text {adh }}$ are shown in Figure 2C. We observed a maximum of the adhesion in weakly acidic solution. The observed dependence compares qualitatively well with the trend of the percentage of LM cells attached to Cytodex [5], which also reaches a maximum as a function of $\mathrm{pH}$. On the other hand, 
the membrane-glass force measured by Priel et al. [16] was monotonically decreasing with $\mathrm{pH}$ increasing from 4 to 10 .

A maximum of $\sigma_{\mathrm{adh}}$ as a function of $\mathrm{pH}$ may occur between the two points of zero charge, $\mathrm{pH}_{\mathrm{pzc}}^{\mathrm{G}}$ and $\mathrm{pH}_{\mathrm{pzc}}^{\mathrm{M}}$, of the glass and the membrane (as explained qualitatively in S5). Note that in a very thin asymmetric film the points of zero charge of the two surfaces can be expected to be shifted by 1-2 units compared to the free glass and membrane surface. The reason is that the membrane surface at $\mathrm{pH}_{\mathrm{pzc}}^{\mathrm{M}}$ has zero charge, but due to the proximity of the negatively charged glass, its potential $\phi^{\mathrm{M}}$ is negative instead of zero, and is of the order of the potential of the free glass surface [15]. The magnitude of this effect is estimated in S5.

The values of $\sigma_{\text {adh }}$ obtained here are of the same order of magnitude as the one obtained with the dynamic method described in Ref. [15] and agree with the theoretical estimate given there. The previous value $\left(\sim 0.2 \mathrm{~mJ} / \mathrm{m}^{2}\right.$ for azolectin membrane containing some protein) is about 3 times lower than $\sigma_{\text {adh }}$ measured here at the same ionic strength in the absence of the protein. This can be the result of the electrostatic repulsion between the glass and the negatively charged $\mathrm{MscS}$ protein used in Ref. [15]. Indeed, in accord with this conclusion, the membrane viscosity in Fig. 5 of [15] decreases with the addition of proteins, indicating that the membrane is unsticking from the glass. However, another possible explanation of this difference is dynamic complications with the method used in Ref. [15], and the neglected dependence of $k_{\eta}$ on $v_{L}$ in particular. Therefore, this conclusion must be considered with care.

In conclusion, the adhesion energy of a lipid bilayer to the patch-clamp pipette can be measured with good precision using fluorescent microscopy for determination of the immobilization point upon mechanical compensation of the capillary force acting on a patch. The method is relatively fast, reproducible and yields results that compare well with previous experimental and theoretical estimates. The main limitation of the precision with which this method can be used is the precision with which $\Delta p$ can be controlled; however, this can be significantly improved. To our knowledge, our study describes for the first time truly static method for measurement of the adhesion free energy between the lipid bilayer and the glass of the patch pipette. This technique can be potentially applied to study quantitatively the adhesion of model bilayers and real cells to glass. In real cells, the properties of the bilayer will be of secondary importance to those of the glycocalyx and the membrane proteins. The comparison of $\sigma_{\text {adh }}$ of the azolectin bilayers studied here with $\sigma_{\text {adh }}$ of bilayers containing proteins reported in [15] indicates significant electrostatic effects due to the 
proteins. Numerical data for $\sigma_{\text {adh }}$ of glycocalyx-mediated adhesion will undoubtedly be a valuable source of information about the related strong non-DLVO interactions (attractive hydrogen bonds and polymer brush osmotic repulsion). But the most interesting object to study with the proposed method is the specialized membrane "adhesive" proteins, the integrins, especially in view of their role in the physiology of mechanotransduction [24] and pathology of cancer progression [25].

\section{Materials \& Methods}

Liposomes consisting of azolectin (99.9\%) and rhodamine-PE (0.1\%) were prepared by the $\mathrm{D} / \mathrm{R}$ method [26]. $2 \mathrm{mg}$ of the mixture of azolectin and rhodamine-PE were dissolved in chloroform, and dried under a stream of nitrogen to make lipid films. $1 \mathrm{~mL}$ of D/R buffer $(100-500 \mathrm{mM} \mathrm{KCl}$, $5 \mathrm{mM}$ HEPES-KOH, $40 \mathrm{mM} \mathrm{MgCl}_{2}$, and $\mathrm{HCl}$ ) was added. The $\mathrm{MgCl}_{2}$ is needed since $\mathrm{Mg}^{2+}$ saltbridges between the glass and the membrane promote formation of and stabilize the gigaseal. In the absence of $\mathrm{HCl}, \mathrm{pH}$ was 7.2 ; we used $\mathrm{HCl}$ to adjust $\mathrm{pH}$ to 4.0 or 5.5 as determined by a $\mathrm{pH}$ meter (SevenEasy; METTLER TOLEDO). The lipid film was sonicated to form a liposome suspension that was transferred into a $15-\mathrm{mL}$ falcon tube where further $2 \mathrm{~mL}$ of $\mathrm{D} / \mathrm{R}$ buffer were added. After an incubation period of 2-3 hours, the solution was centrifuged at 250,000 $\times g$. The pellet was collected and resuspended in $60 \mu \mathrm{L}$ of $\mathrm{D} / \mathrm{R}$ buffer, then spotted onto a microscope slide and dehydrated under vacuum overnight at $25^{\circ} \mathrm{C}$. The dried lipid films were rehydrated with $\mathrm{D} / \mathrm{R}$ buffer for $3 \mathrm{~h}$ at $4{ }^{\circ} \mathrm{C}$. An aliquot of liposomes $(5 \mu \mathrm{L})$ was placed on the bottom of the recording chamber. Unilamellar blisters emerged from the collapsed liposomes after $30 \mathrm{~min}[26,27]$. Borosilicate glass pipettes (Drummond Scientific) were pulled using a Flaming/Brown pipette puller (P-87; Sutter Instruments). Glass pipette of bubble number 4.0-5.0 were used for the patch fluorescence microscopy. The fluorescence images of liposome membrane were recorded in the inside-out excised configuration (similar to those in Ref. [23]) without applying voltage; Zeiss LSM 700 confocal microscope was used, with a long working distance water immersion objective (63×; NA 1.15; Carl Zeiss). A 555-nm laser line was used to excite the Rhodamine labeled patches with emission detected using a long pass 560-nm filter. To visualize liposome patches the pipette tip was bent to $\sim 30^{\circ}$ with a microforge (Narishige; MF-900) to become parallel with the bottom face of the chamber. The same saline solution was used for both pipette and bath solution.

The gigaseal formation was confirmed electrically using the resistance mode of the patch-clamp system (Molecular Devices; pCLAMP10 software). The patch membrane was observed under the 
confocal microscope at distances from the tip of the pipettes in the range $L=10-25 \mu \mathrm{m}$. The pressure $\Delta p$ applied to patch pipettes was controlled by a High Speed Pressure Clamp-1 apparatus (HSPC-1; ALA Scientific Instruments) and was monitored using a piezoelectric pressure transducer (Omega Engineering). In the absence of applied pressure, the patch was creeping up the pipette. Progressively increasing positive pressure was applied to the patch pipette until the capillary force was compensated at $\Delta p_{\text {stop }}$, Eq. (3), and the liposome patch membrane creeping stopped. The immobilization point was identified via the absence of significant shift of the patch for 2-3 min; in addition, the patch dome was flat, cf. Figure 2A. The pressure was further increased above $\Delta p_{\text {stop }}$ to confirm that the immobilization point was reached (at $\Delta p>\Delta p_{\text {stop }}$, the dome bended inward and backward creep was observed). Each measurement was repeated with 2-3 independent liposomes and pipettes with different radius and opening angle. The pipette shape or length $L$ did not affect the measurement. With HSPC-1, the pressure can be controlled with precision of \pm 133 $\mathrm{Pa}$ (i.e. $\pm 1 \mathrm{mmHg}$ ). The change of $\Delta p$ with the minimal possible step, $\pm 133 \mathrm{~Pa}$, leads to visible change in the creep velocity, which means that the precision of determination of $\Delta p_{\text {stop }}$ is also \pm $133 \mathrm{~Pa}$. The value of $R_{\mathrm{c}}$ was determined from the image of the immobilized patch with accuracy of $\pm 0.1 \mu \mathrm{m}$ using the measurement tool of the ZEN imaging software (Carl Zeiss). Thus, the precision of determining $\Delta p$ presents the main limitation for the precise calculation of $\sigma_{\text {adh }}$ : according to Eq. (3), the precision of the measured $\sigma_{\mathrm{adh}}$ is $\pm 133 \times R_{\mathrm{c}} / 2 \sim \pm 0.1-0.2 \mathrm{~mJ} / \mathrm{m}^{2}$.

\section{Acknowledgements}

We would like to thank Prof. Frederick Sachs for his critical comments and suggestions. R.S. is grateful for funding by Bulgarian National Science Fund through Contract 51 from 12.04.2016 with Sofia University, N.B. has been supported by an International Scholarship from the University of New South Wales (UIPA) and B.M. is recipient of a Principal Research Fellowship from the National Health and Medical Research Council of Australia.

\section{Supporting information}

S1. List of symbols and abbreviations. S2. Young's equation for the adhered patch. S3. Experimental data. S4. Electrostatic repulsion in very thin films. S5. Effect of the $\mathrm{pH}$ and shift of the point of zero charge with thickness. 


\section{References}

1. Zhu C.; Bao G.; Wang N. Cell mechanics: Mechanical Response, Cell Adhesion, and Molecular Deformation. Annu. Rev. Biomed. Eng. 2000 2, 189-226.

2. Hori K. Adhesion of Bacteria. In: Biofilm and material science. 2015 Ch. 4.

3. Curtis A.S.G.; Forrester J.V.; McInnes C.; Lawrie F. Adhesion of Cells to Polystyrene Surfaces. J. Cell Biol. 1983 97, 1500-1506.

4. Owens N.F.; Gingell D.; Rutter P.R. Inhibition of Cell Adhesion by a Synthetic Polymer Adsorbed to Glass Shown under Defined Hydrodynamic Stress. J. Cell Science 1987 87, 667675 .

5. Gotlib L.J. Isolation of Cell Plasma Membranes on Microcarrier Culture Beads. Biochim. Biophys. Acta 1982 685, 21-26.

6. Jacobson B.S. Improved Method for Isolation of Plasma Membrane on Cationic Beads. Biochim. Biophys. Acta 1980 600, 769-780.

7. Suchyna, T.M.; Markin, V.S.; Sachs, F. Biophysics and Structure of the Patch and the Gigaseal. Biophys. J. 2009 97, 738-747.

8. Suchyna T.M.; Sachs F. Mechanosensitive Channel Properties and Membrane Mechanics in Mouse Dystrophic Myotubes. J. Physiol 2007 581, 369-387.

9. Nomura T.; Cox C.D.; Bavi N.; Sokabe M.; Martinac B. Unidirectional Incorporation of a Bacterial Mechanosensitive Channel into Liposomal Membranes. FASEB J. 2015 29, 43344345

10. Ursell, T.; Agrawal, A.; Phillips, R. Lipid Bilayer Mechanics in a Pipette with Glass-bilayer Adhesion. Biophys. J. 2011 101, 1913-1920.

11. Absolom D.R.; Lamberti F.V.; Policova Z.; Zingg W.; van Oss C.V.; Neumann A.W. Surface Thermodynamics of Bacterial Adhesion. Appl. Env. Microbiol. 1983 46, 90-97

12. Blake, T.D., in: C. John, Bos (Eds.), Wettability, Surfactant Science Series, vol. 49, 1993, p. 291-309.

13. Martic G.; De Coninck J.; Blake T.D. Influence of the Dynamic Contact Angle on the Characterization of Porous Media. J. Colloid Interface Sci., 2003 263, 213-216.

14. Smith, A.S.; Sackmann, E.; Seifert, U. Pulling Tethers from Adhered Vesicles. Phys. Rev. Lett. 2004 92, 208101. 
15. Slavchov R.I.; Nomura T.; Martinac B.; Sokabe M.; Sachs F.,Gegaseal Mechanics: Creep of the Gigaseal under the Action of Pressure, Adhesion and Voltage. J. Phys. Chem. B. 2014 $118,12660-12672$.

16. Priel A.; Gil Z.; Moy V.T.; Magleby K.L.; Silberberg S.D. Ionic Requirements for Membrane-glass Adhesion and Gigaseal Formation in Patch-clamp Recording. Biophys. J. 2007 92, 3893-3900.

17. Opsahl L.R.; Webb W.W. Lipid-glass Adhesion in Giga-sealed Patch-clamped Membranes. Biophys. J. 1994 66, 75-79.

18. Jerison E.R.; Xu Y.; Wilen L.A.; Dufresne E.R. Deformation of an Elastic Substrate by a Three-phase Contact Line. Phys. Rev. Lett. 2011 106, 186103.

19. Churaev N.V.; Starov V.M.; Derjaguin B.V. The Shape of the Transition Zone between a Thin Film and Bulk Liquid and the Line Tension. J. Colloid Interface Sci. 1982 89, 16-24

20. Nomura T.; Cranfield C.G.; Deplazes E.; Owen D.M.; Macmillan A.; Battle A.R.; Constantine M.; Sokabe M.; Martinac B. Differential Effects of Lipids and Lyso-Lipids on the Mechanosensitivity of the Mechanosensitive Channels MscL and MscS. PNAS. 2012 109, 8770-8775.

21. Petrache H.I.; Zemb T.; Belloni L.; Parsegian V.A. Salt Screening and Specific Ion Adsorption Determine Neutral-Lipid Membrane Interactions. PNAS 2006 103, 7982-7987.

22. Cremer P.S.; Boxer S.G. Formation and Spreading of Lipid Bilayers on Planar Glass Supports. J. Phys. Chem. B 1999 103, 2554-2559.

23. Bavi N.; Nakayama Y.; Bavi O.; Cox C.D.; Qin Q.H.; Martinac B. Biophysical Implications of Lipid Bilayer Rheometry for Mechanosensitive Channels. PNAS 2014 111, 13864-13869.

24. Ingber, D.E. Tensegrity and Mechanotransduction. J. Bodyw. Mov. Ther. 2008 12, 198 200

25. L. Seguin, J.S. Desgrosellier, S.M. Weis, D.A. Cheresh, Integrins and Cancer: Regulators of Cancer Stemness, Metastasis, and Drug Resistance. Trends Cell Biology 2015 25, 234-240

26. Häse C.C.; Le Dain A.C.; Martinac B. Purification and Functional Reconstitution of the Recombinant Large Mechanosensitive Ion Channel (MscL) of Escherichia coli. J. Biol. Chem. $1995270,18329-18334$. 
27. Delcour A.H.; Martinac B.; Adler J.; Kung C. Modified Reconstitution Method Used in Patch-clamp Studies of Escherichia coli Ion Channels. Biophys. J. 1989 56, 631-636. 